\title{
Kardiovaskuläre Prävention als Technik zur Bildung von Leben selbst ${ }^{1}$
}

\author{
Eine ethnographische Untersuchung.
}

JÖRG NIEWÖHNER \& MICHALIS KONTOPODIS

»[W]enn die Biologie [im 18. Jahrhundert] unbekannt war, gab es dafür einen einfachen Grund: das Leben selbst existierte nicht.« (Foucault 1974: 168) Leben selbst entsteht laut Michel Foucault mit der Entwicklung eines epistemischen Systems, das die grundsätzliche Vernetztheit aller lebenden Dinge postuliert. Diese Vernetztheit wiederum produziert die Bedingungen, die die Entstehung des Konzepts von Leben selbst befördern (Franklin 2000: 193). Leben selbst bezeichnet also die Gesamtheit der lebendigen Qualitäten und Kapazitäten. Zu Beginn des 21. Jahrhunderts diagnostiziert Sarah Franklin in ihrer Analyse der Entwicklungen der neuen Genetik die signifikante Technologisierung und Kapitalisierung dieses Lebens selbst. Die Lebenswissenschaften zielten in der Logik und Praxis ihrer Interventionen nicht mehr nur noch auf Gesundheit, Tot und Krankheit sondern eben auf Leben selbst. Nikolas Rose führt diese Analyse fort und markiert in seiner Kartographie der Gegenwart fünf charakteristische »Mutationen « oder Dimensionen einer Politik des Lebens selbst: Molekularisierung, Optimierung, Subjektivierung, somatische Erfahrung und economies of vitality (vgl. Rose 2007: 4ff.).

1 Wir übersetzen das Konzept life itself, wie es Sarah Franklin und Nikolas Rose in den letzten Jahren etabliert haben, als Leben selbst, um es klar von möglicher Weise ähnlich lautenden Konzepten wie Lebenspolitik oder Vitalpolitik abzugrenzen. 
Molekularisierung schreibt den Trend der Biomedikalisierung, d.h. die Transformation der modernen Medizin durch das Aufkommen von technoscience, fort (Clarke, et al. 2003) und diagnostiziert eine zunehmende Verortung von Bedeutung auf molekularer Ebene. Optimierung markiert die zunehmende Verwischung der Unterscheidung von Risiko bzw. Suszeptibilität und Verbesserung (enhancement). Subjektivierung befasst sich mit der Verschiebung weg von pastoralen Herrschaftsformen zu neuen Formen der biopolitischen Aktivierung. Somatische Erfahrung meint die zunehmend zentrale Rolle von biologisch gewusster, erfahrener und produzierter Körperlichkeit in den Konstitutionsprozessen von Sozialität und Individualität. Economies of vitality bezieht sich auf die globale Kapitalisierung von Leben selbst durch das rasante Wachstum von Gesundheitsmärkten und ihrer zunehmenden Vernetzung zwischen staatlichen, halb-staatlichen, supra-nationalen und privaten Akteuren. Diese fünf Mutationen werden vor allen Dingen durch Analysen der neueren genetischen Technologien und ihren Anwendungen herausgearbeitet.

In diesem Beitrag stellen wir die Frage inwiefern diese Diagnose nur auf aktuelle genetische Anwendungen zutrifft oder ob sie auch die Entwicklungen im Feld der komplexen, chronischen Krankheiten angemessen charakterisiert. Dies ist insofern von besonderer Relevanz, als dass chronische Erkrankungen nicht nur in westlichen Gesundheitssystemen den bei weitem wichtigsten Posten darstellen. Wir untersuchen diese Frage am Beispiel der Herzkreislauferkrankungen und Übergewicht, speziell dem zunehmenden Trend hin zur Prävention. Die Genetik spielt in diesem Feld nicht die herausragende Rolle, wie sie es vielleicht in den Bereichen Klonen, Reproduktionsmedizin oder Gendiagnostik tut. Trotzdem durchläuft die biomedizinische Forschung zur Ätiologie von Herzkreislauferkrankungen eine ähnliche Entwicklung. Die Spezifika dieser Entwicklung und ihre Auswirkungen auf die Praxis von Herzkreislaufprävention wollen wir im Folgenden skizzieren. Wir tun dies anhand einer detaillierten Diskussion der ersten drei Roseschen Mutationen ${ }^{2}$ im Bereich der Herzkreislaufprävention mit einem Fokus auf Deutschland - Molekularisierung, Optimierung, Subjektivierung - und berücksichtigen dabei auch die wichtigen Verschränkungen zwischen Medizin und Ökonomie. Anhand

2 Die Mutationen vier und fünf - Somatische Erfahrung und Economies of Vitality - können wir auf der Basis unserer empirischen Befunde derzeit nur unzureichend erörtern und klammern sie daher in diesem Aufsatz aus. 
eines ethnographischen Beispiels von Präventionsprogrammen in einem Berliner Kindergarten diskutieren wir die konkreten Auswirkungen dieser Mutationen, so wie wir sie für die Konzeption kardiovaskulärer Krankheiten aufzeigen, auf Präventionspraxis. Wir zeigen damit, dass die Analysen auf der Basis von »modest empiricism« (Rabinow/Rose 2006) ${ }^{3}$, wie wir sie in den ersten drei Schritten durchführen, sinnvoller Weise durch ethnographisches Material von konkreten Praxisformen begleitet werden können, um zu vermeiden, dass die Auswirkungen von biopolitischen Herrschaftsformen in spezifischen Alltagen unterbelichtet werden.

Unsere Analyse baut auf Ergebnissen eines interdisziplinäres Forschungsprojekts auf, in dessen Rahmen wir mittels historischer Analyse, Diskurs- und Inhaltsanalysen von grauer Literatur und Fachpublikationen, qualitativer Analysen von Arzt-Patienten Gespräche, Interviews mit Forschenden in der Wissenschaft und Politiker/innen und ethnographischer Feldforschung in Hausarztpraxen, Kliniken und Kindergärten im Entstehen begriffene Konstellationen von Herzkreislaufprävention untersucht haben. ${ }^{4}$ Die Ergebnisse dieser Untersuchung können hier nicht vollständig präsentiert werden. ${ }^{5}$ Im Kontext der hier dargestellten Argumentation werden wir auf Diskursanalysen und ethnographische Materialien Bezug nehmen und an diesen Materialien exemplarisch eine breitere Entwicklung diskutieren. Die ethnographische Untersuchung von Kindergärten hat hauptsächlich in verschiedenen Kindergärten Berlins stattgefunden. ${ }^{6}$ Das spezifische Beispiel im letzten Abschnitt dieses Aufsatzes stammt aus einem Kindergarten in

3 Rabinow und Rose grenzen ihr Konzept des gemäßigten Empirismus von philosophischen Analyse ab und betonen damit ihre Achtsamkeit gegenüber Verschiebungen, Unstimmigkeiten und Auffälligkeiten in Diskursen und Praxen.

4 Das Forschungsprojekt wurde gefördert aus Mitteln des deutschen Bundesministeriums für Bildung und Forschung (01GWS051-054). Wir danken herzlich unseren Kooperationspartnern Regine Kollek und Martin Döring (Universität Hamburg), Jeanette Madarász und Martin Lengwiler (WZB und Universität Basel), Vittoria Braun und Christoph Heintze (Charité Berlin) und Katrin Amelang, Tom Mathar und Stefan Beck (Humboldt-Universität zu Berlin).

5 Eine ausführlichere Darstellung der Ergebnisse findet sich in (Niewöhner 2011)

6 In einem vergleichenden Ansatz, dessen Ergebnisse hier nicht präsentiert werden können, wurden verschiedene Kindergärten in Bezirken Berlins und New Yorks verglichen (Kontopodis 2009). 
einem sozial schwachen Viertel im ehemaligen Ostberlin, der seit geraumer Zeit an dem Präventionsprogramm >Tigerkids< teilnimmt. Dies ist ein Programm einer gesetzlichen Krankenversicherung, das bundesweit eingesetzt wird und in unabhängigen Evaluationen sehr positiv abschneidet.

\section{MOLEKULARISIERUNG}

Mit der erfolgreichen Eindämmung der infektiösen Erkrankungen zu Beginn des 20. Jahrhunderts rücken die chronischen Erkrankungen in den Mittelpunkt des öffentlichen Gesundheitsinteresses: allen voran Krebs und Herzkreislauferkrankungen (Lengwiler/Beck 2008). Letztere, und hier speziell Übergewicht und erhöhter Blutdruck, werden von Beginn an als multifaktorielles Krankheitsbild diskutiert. Die vermuteten Ursachen reichen von eher materiellen Faktoren, wie übermäßige Energiezufuhr oder mangelnde Bewegung, bis hin zur >modernen Zivilisation< mit ihren freien und liberalen Lebensgewohnheiten und ihrer raschen Entwicklung und Veränderung (z.B. Fisk 1915). Nach dem Zweiten Weltkrieg beginnt mit der Seven Countries und der Framingham Studie (Keys, et al. 1950, Kannel/McGee 1979) der rasante Aufstieg der Risikofaktorepidemiologie. Auf der Basis epidemiologischer Daten werden damit Rauchen, Alter, Geschlecht, Blutdruck und Cholesterin zu den einflussstärksten Risikofaktoren für kardiovaskuläre Erkrankungen. Die genaue Rolle von Übergewicht in dieser Gemengelage von Faktoren bleibt bis heute umstritten. Ein wenig abseits dieser Liste spielt aber auch Stress weiterhin eine wichtige Rolle; in den 1960er und 1970er Jahren nicht mehr als »rasche Entwicklung«, sondern als Typ A Verhalten bzw. in Deutschland als Managerkrankheit. (Lengwiler/Madarász 2010)

Im Einklang mit einer zunehmenden Molekularisierung der Biologie und mit ihr einer Biomedikalisierung der Medizin (Clarke, et al. 2003), beginnt in den 1980er Jahren eine Molekularisierung dieser Risikofaktoren, die bis heute andauert. Beispielhaft für diese Veränderung steht das Aufkommen des »metabolischen Syndroms« (vgl. Reaven 1988). Das metabolische Syndrom bezeichnet eine Reihe physiologischer Veränderungen - zentrale Adipositas, Bluthochdruck, Dyslipidaemie und erhöhte Nüchternglukose - deren gemeinsames Auftreten statistisch signifikant mit Herzkreislaufereignissen korreliert. Als 
statistisches bzw. epidemiologisches Phänomen stellt das metabolische Syndrom zum einen eine Ausdifferenzierung und Erweiterung einiger Risikofaktoren dar. Zum anderen verweist dieses Syndrom bereits auf die Interdependenz dieser Faktoren. Diesem in ersten Ansätzen systemischeren Verständnis soll in der hausärztlichen Praxis dadurch Rechnung getragen werden, dass Risikofaktoren nicht isoliert betrachtet werden, sondern die Diagnose eines Faktors automatisch die Untersuchung weiterer Parameter nach sich zieht. Allerdings sind sowohl der ontologische Status sowie der klinische Nutzen des Metabolischen Syndroms von Anfang an und bis heute heftig umstritten (vgl. Kahn, et al. 2005). Die Ursache für diesen Streit liegt vor allem in der mangelnden mechanistischen Erklärbarkeit dieses statistischen Phänomens. Die (gemeinsame) physiologische Basis für diese Häufung von Stoffwechselveränderungen ist bis heute nicht hinreichend verstanden. Die Suche nach der Antwort hat in den letzten zwei Jahrzehnten eine massive Molekularisierung des ätiologischen Modells von Herzkreislauferkrankungen mit sich gebracht. Dies ist nicht der Ort, um diese enorm vielfältige Forschungslandschaft näher zu skizzieren. Stattdessen konzentrieren wir uns auf einen einzelnen wichtigen Strang, der exemplarisch verdeutlicht, auf welche Art und Weise sich Risikofaktoren im Zuge dieser Forschung verändern.

Folgt man einer in der Biomedizin weit verbreiteten Meinung, so lässt sich in der aktuellen Forschung eine direkte Verbindung zwischen dem, was in der Forschung gemeinhin als chronischer Stress verstanden wird und erhöhtem Herzkreislaufrisiko. In aller Kürze: chronischer Stress, z.B. durch eine Überforderung am Arbeitsplatz oder eine unglückliche Ehe, resultiert in einer permanenten Aktivierung der Stressachse, d.h. zu einer vermehrten Ausschüttung des Hormons Cortisol. Eine derartige Hypercortisolaemie verursacht mit der Zeit eine Insulinresistenz, die wiederum in direktem Zusammenhang mit Verschiebungen des Metabolismus und damit erhöhtem Herzkreislaufrisiko steht. Hier ist es also in den letzten zehn Jahren gelungen, einen Diskurs und eine Forschungsplattform zu etablieren, die einen molekularen Mechanismus für den Zusammenhang zwischen chronischem Stress und Herzkreislaufrisiko plausibel darstellen kann. ${ }^{7}$

7 An dieser Stelle können wir nicht auf die Vorannahmen und Kontingenzen eingehen, die die Produktion dieser Befunde notwendig beinhaltet. (Niewöhner 2008) 
Diese Entwicklung steht exemplarisch für eine Molekularisierung und Vernetzung von Risikofaktoren im Bereich der Herzkreislauferkrankungen. Im Vergleich zu Blutdruck oder Cholesterinwerten stellen die Hoch- oder Herunterregulierung von funktionalen Hirnarealen oder hormonellen Pfaden deutlich dynamischere und systemischere Messgrößen dar. Genetische Komponenten werden in einer solchen molekularen Sicht von Metabolismus einerseits zwar sichtbarer, denn ein einzelner Rezeptor ist in seinen genetischen Komponenten besser $\mathrm{zu}$ untersuchen, als ein Parameter wie Blutdruck, der wiederum von vielen Faktoren bestimmt wird. Andererseits jedoch verweisen diese Netzwerke auf Vielfältigkeit und Dynamik. Gene, konzipiert als statische DNA Sequenzabschnitte, sind in dieser post-genomischen Sicht auf Metabolismus nur in den wenigen Fällen noch von Bedeutung, in denen Mutationen tatsächlich gravierende Auswirkungen auf den Phänotyp haben. Vielmehr werden Gene bzw. wird DNA Sequenz zunehmend eingebettet in Analysen von Genexpression und ihrer Regulierung über genomische Kontexte und epigenetische Faktoren. Epigenetische Faktoren, d.h. die chemische Veränderung von DNA mit Folgen für ihre Ablesbarkeit, rücken die Regulierung von Genexpression durch zelluläre Umwelt in den Fokus; wobei zelluläre Umwelt sowohl andere Zellen als auch den Organismus an sich samt seiner sozialen und materiellen Umwelt bezeichnet. Was epidemiologische Studien bereits angebahnt hatten, wird auf der Basis von Epigenetik nun auch molekular nachvollziehbar. So führt beispielsweise eine spezifische Ernährung von trächtigen Mäusen zur Herunterregulierung eines Gens in der Nachkommenschaft, das für die Fellfarbe mitverantwortlich ist. Die Nachkommen verändern daher unter diesem Ernährungsregime ihre Fellfarbe im Vergleich zur Mutter (Waterland/Jirtle 2004). Ähnliche Effekte lassen sich durch gezielte Veränderungen in materieller und sozialer Umwelt der Tiere wie auch beim Menschen erzielen bzw. nachweisen (z.B. McGowan, et al. 2009, Szyf, et al. 2008, Weaver, et al. 2004).

Neben diesen Effekten der sozialen und materiellen Umwelt auf Genexpression und damit Phänotyp und Physiologie, sind durch das Voranschreiten biologischen Wissens über epigenetische Prozesse auch zeitliche Dimensionen für Entwicklung und Krankheit auf neue Weise relevant geworden. Unter der Hypothese »Entwicklungsbiologische Ursachen für Krankheiten im Erwachsenenalter « (developmental origins of adult disease, DOAD) werden zunächst die prä-, peri- und 
neonatalen, prägenden Einflüsse auf den adulten Stoffwechsel zunehmend besser verstanden (Gluckman, et al. 2005). Stress und Überoder Unterernährung während spezifischer Phasen vor, während und nach der Schwangerschaft scheinen einen stärkeren Einfluss auf Stellschrauben zu haben, die später für Reaktionen auf ungünstige Ernährung oder chronischen Stress verantwortlich sind, als bisher angenommen. Hinzu kommt, dass epigenetische Mechanismen es auch wahrscheinlicher werden lassen, dass solche Prägungseffekte unter Umgehung der DNA Sequenz, d.h. ohne Mutation, an die nächste Generation weitergegeben werden (z.B. Roth, et al. 2009). Auch hier werden also zunehmend molekulare Mechanismen plausibilisiert, um zu erklären, wie es passieren kann, dass die Enkelkinder von holländischen Großeltern, die im Zweiten Weltkrieg während der Schwangerschaft einer Hungersnot ausgesetzt waren, als Erwachsene 50 Jahre später ein erhöhtes Diabetesrisiko haben (Roseboom, et al. 2006).

Das biomedizinische Verständnis von Krankheit bzw. Krankheitsrisiko wird also zunehmend auf einer molekularen Ebene abgebildet, und es wird versucht, Krankheit über molekulare Vorgänge zu erklären. Dies führt allerdings überraschender Weise nicht zu einem rein molekular-deterministischen bzw. techno-somatischen Körperbild (Pickersgill 2009). Statt eines hermetischen Körpers, der durch die Haut begrenzt als geschlossenes, selbst-referentielles System agiert, öffnet in einer gegenläufigen Entwicklung gerade die Molekularisierung von Körper diesen epistemologisch für seine materielle und soziale Umwelt sowie für seine eigene Vergangenheit. Das autonome Individuum bleibt in der Medizin zwar als medizinethisches Konstrukt und Fokus praktischen Handelns präsent. In der Grundlagenforschung jedoch entsteht ein neues Bild: das Bild von einem vielfältig eingebetteten Individuum, dessen Neigungen und Vorlieben, Krankheiten und Talente hochgradig durch seine Umwelten und seine eigene Vergangenheit geprägt sind. Dies schließt zwar Plastizität und Reaktionsfähigkeit auf Umweltveränderungen nicht aus. Prägung und Umwelteinfluss geben jedoch in diesem Bild bestimmte Spannweiten vor, innerhalb derer agiert werden kann. Ein Ausbrechen aus diesen Vorgaben erfordert einen erhöhten Aufwand. Die Molekularisierung des Verständnisses von Krankheit führt hier also zu einem Körperbild, das zwar stärker molekularbiologisch geprägt ist. Dieses Verständnis ist allerdings nicht eines, das mit Manipulierbarkeit und Wahlmöglichkeiten im direkten und individualistischen Sinne von Rose einhergeht 
(Rose 2007). Die adulte Biologie des eigenen Körpers wird durch diese Verschiebungen weniger verfügbar, stärker biologisiert und damit auch reifiziert. Intervenier- und Manipulierbarkeit des individuellen Körpers müssen vermehrt über die materielle und soziale Umwelt, über Bildung und Entwicklung und über verschiedene Zeithorizonte, v.a. Generationen, hinweg vermittelt werden.

\section{OPtimiERUNG}

Für Herzkreislauferkrankungen lässt sich also eine Molekularisierung bei gleichzeitiger Öffnung des Körperkonzepts diagnostizieren. Wie sieht es mit Roses zweiter Mutation - Optimierung - aus? Mit Blick auf die Entwicklungen in der neuen Genetik hat Sarah Franklin konstatiert, dass wir nun ein Zeitalter biologischer Kontrolle erreicht haben. Sie, wie auch andere, sehen eine Konfiguration entstehen, in der das Biologische nicht mehr als gegeben und begrenzend wahrgenommen wird. Stattdessen markiert das Gebiet des Biologischen zunehmend einen gänzlich kontingenten Zustand. Körper wird manipulierbar und Medizin hat nicht nur die Aufgabe einen durch Krankheit verlorenen >natürlichen< Zustand wieder herzustellen. Die Normativität dieser natürlichen Gesundheit verblasst im Angesicht technologischer Möglichkeiten: »Biotechnologie verändert, was es heißt, biologisch $\mathrm{zu}$ sein.«(Rose 2007: 17)

Diese Entwicklung ist im Bereich der Herzkreislauferkrankungen so nicht nachzuvollziehen. Zwar können wir eine Molekularisierung der ätiologischen Modelle konstatieren. Auch stellen wir fest, dass das oben analysierte neue Körperkonzept dazu führt, dass Medizin sich nicht nur mit dem Körper im engeren Sinne befasst, sondern im Sinne einer Technologie des Lebens eine große Vielzahl an Parametern zu kontrollieren sucht. Hierzu stehen der Medizin auch eine Reihe weiterer Akteure aus Politik, Sozialarbeit und Pädagogik zur Seite. Allerdings beschränkt sich dieses neue Regime auf immer detailliertere Diagnostik. Jeder Bereich des Lebens wird unter Vulnerabilitätsgesichtspunkten erfasst und analysiert: von körperlichen Voraussetzungen, über das soziale Umfeld und Alltagsgewohnheiten. Jeder Winkel des Lebens leistet einen Beitrag $\mathrm{zu}$ einem Risikoscore und $\mathrm{zu}$ einer erhöhten bzw. gesenkten Vulnerabilität. In diesem Sinne ist Herzkreislaufmedizin und -prävention selbstverständlich eine Technologie des 
Lebens selbst. Sie versucht Leben umfassend zu taxieren und nach Vulnerabilitätsgesichtspunkten zu ordnen.

Dies führt aber derzeit nicht dazu, dass das Gebiet des Biologischen kontingenter oder verfügbarer wird. Das Wissen, das hier neu produziert wird, hat in der Tat hauptsächlich taxierende und ordnende Qualitäten. Körper werden daher anders, manche würden sagen >umfassender< taxiert. Sie werden detaillierter repräsentiert, ohne dass daraus neue Interventionen folgen würden. Dabei liegt den Technologien des Lebens ein durch und durch moderner Wissensbegriff $\mathrm{zu}$ Grunde, der nicht nur Repräsentation, sondern immer auch Intervention bezeichnet (Hacking 1983). Gewusst ist etwas erst, wenn in es eingegriffen werden kann: seien dies Körper, Maschinen oder Software. Medikamentöse Eingriffe allerdings beschränken sich im Bereich der Herzkreislauferkrankungen auf die weit verbreiteten Lipidund Blutdrucksenker. Wirksame Medikamente, die langfristig und ohne signifikante Nebenwirkungen den Energiehaushalt einpegeln und damit Gewicht senken oder stabilisieren könnten, existieren derzeit nicht. Appetitkontrolle, verminderte Energieaufnahme aus der Nahrung, eine Regulierung des Belohnungssystems usw. - alle Versuche in diese hochgradig redundant organisierten Systeme einzugreifen, haben bisher nicht zufrieden stellend funktioniert. Von einer Manipulierbarkeit des Biologischen kann daher keine Rede sein. Im Gegenteil: gerade die biologischen Grundlagenforscher in Deutschland sprechen zunehmend dem Individuum, im Sinne eines autonomen Subjekts, die Fähigkeit ab, seine bzw. ihre Biologie selbstbestimmt zu verändern. Prägungseffekten einerseits und Selbsterhaltungsmechanismen von Fettzellen bzw. Stoffwechselmustern andererseits wird eine derartige Stabilität zugeschrieben, dass man Individuen nicht zutraut, sich aus diesen Mustern zu befreien. So verändert zwar die Molekularbiologie auch in diesem Feld, was es heißt, biologisch zu sein. Sie tut dies allerdings gerade nicht in Richtung größerer Gestaltungsmöglichkeiten, sondern in Richtung größerer Widerständigkeit und Starrheit.

Dass diese Form der biologischen Widerständigkeit von der Forschung propagiert wird, ist in der modernen Landschaft der molekularen Biomedizin neu. Bisher wurde hier mit einer gewissen Nonchalance Übergewicht als Lebensstil bedingt und damit offen für Intervention konzipiert. Dieses Bild wandelt sich nun und kommt damit dem Verständnis näher, dass unter PraktikerInnen in der Versorgung und Prävention schon lange bekannt ist. Dort wo Patienten mit samt ihrer 
Lebenswelt Rat suchen, war schon immer offensichtlich, dass sich elementare Dinge des Alltags, wie Essen und Bewegen, nicht einfach durch punktuelle Interventionen verändern lassen. Dies wird von HausärztInnen und PräventionsmedizinerInnen nicht rein biologisch begründet. Ihnen wird täglich vor Augen geführt, dass eine Gewichtsabnahme an vielen, aus Forschungsperspektive zunächst vielleicht trivial erscheinenden Dingen scheitert: terminliche Schwierigkeiten bei der Wahrnehmung von Präventionsprogrammen, familiäre Forderungen v.a. an Mütter bei der Auswahl und Zubereitung des Essens, der »innere Schweinehund «, individuell wie sozial wichtige Gewohnheiten und Rhythmen usw. Aus den Erzählungen vieler Ratsuchender und Patient_innen geht ebenfalls hervor, dass Gewichtszunahme nicht nur kontinuierlich verläuft. Stattdessen sind viele Erzählungen gekennzeichnet von Ereignissen, die zu einer rapiden Gewichtszunahme geführt haben: physische Einschränkungen durch orthopädische Eingriffe, Beziehungsprobleme, berufliche Schwierigkeiten usw. Solche Ereignisse führen entweder direkt zu einer schlechteren Energiebalance, z.B. weil Bewegung für einige Zeit nicht möglich ist. Oder sie begünstigen diese indirekt, indem die nötige Disziplin, nicht zu kalorienreich zu essen und sich ein wenig zu bewegen, nicht mehr aufgebracht werden kann. So scheinen, zumindest wenn man den Erzählungen der Betroffenen glauben schenkt, viele >Übergewichtskarrieren< eher stoßweise von Ereignis zu Ereignis zu verlaufen, als kontinuierlich.

Kenntnis und Verständnis dieser Art Faktoren ist meist dem Erfahrungswissen derer vorbehalten, die tagtäglich intensiven Kontakt mit PatientInnen haben und diesen Kontakt auch über einen längeren Zeitraum aufrechterhalten. Dies gilt in Deutschland vor allen Dingen für die Hausärzte, für die klar der Erhalt einer langfristigen Beziehung mit den Patienten an erster Stelle steht; weit vor zu intensiver Beratung oder gar Bewertung von Übergewichtigen, die dadurch vielleicht abgeschreckt werden könnten. Diese Einstellung ändert sich rasch, wenn man den Hausarztsektor verlässt. Bereits in Präventionsprogrammen ist der Kontakt mit Teilnehmerinnen kurzfristiger und stark fokussiert. Hier wird das Umfeld der Teilnehmerinnen schon überwiegend kategorial und häufig psychologisierend wahrgenommen. Gängige Theorien zum Gesundheitsverhalten stehen im Vordergrund. Konzepte wie Motivation, Barrieren, Verhalten und Information werden deutlich häufiger verwendet, als dies bei Hausärzten der Fall ist. Je mehr der 
Kontakt mit den alltäglichen Leben der Menschen abnimmt, desto abstrakter wird die Redeweise über diese Leben. Bereits in der epidemiologischen Forschung sind diese Leben hochgradig klassifiziert, variabilisiert und kontrolliert. Aktuelle biologische Forschung wird in diesen Feldern nicht gezielt genutzt. Wichtig sind hier daher die gängigen Einflussfaktoren auf Übergewicht: sozio-ökonomischer Status inklusive Bildung und Familiensituation, Zugang $\mathrm{zu}$ gesunden Lebensmitteln und Bewegungsmöglichkeiten usw. Zwar stehen hier Populationen im Vordergrund. Das autonome Individuum spielt allerdings als Handlungsträger immer noch eine zentrale Rolle: >man muss den Einzelnen erreichen<, >der Einzelne muss verstehen, wie wichtig es für ihn oder sie ist $<$, >wenn sie es dem Einzelnen nicht klar machen können< usw. Dies sind typische Redewendungen, die zwar die sozialen und materiellen Bedingungen eines einzelnen Lebens in Betracht ziehen, aber trotzdem individuelle, kognitive Faktoren als ausschlaggebend für Verhaltensänderung in den Vordergrund rücken. Sozialität und Alltag sind in dieser Sicht lediglich Kontext für Lebensstile, nicht aber konstitutiv für das Phänomen Übergewicht oder soziales Handeln im Allgemeinen.

Diese Sichtweise beginnt sich nun zu wandeln. In der biologischen Forschung und in der biologischer orientierten Epidemiologie wird die Handlungsträgerschaft weit weniger selbstverständlich dem Individuum zugesprochen. Zwar spielen kognitive Parameter weiter eine Rolle und die Frage nach notwendigem disziplinärem Wissen wird immer noch am häufigsten mit Psychologie beantwortet. Zunehmend aber wird das Individuum mehr oder weniger gänzlich aus der Gleichung herausgenommen. Der biologische Körper in seiner Umwelt wird zum zentralen Gegenstand des Denkens in diesen Feldern. Dementsprechend werden die Interventionen zunehmend weniger als kognitive, wissensvermittelnde Eingriffe gedacht. Hier geht es nicht mehr um Gouvernementalität in dem Sinne, dass man hofft, dem Individuum eine Sorge um sich selbst und Selbstmanagementtechniken näher zu bringen und damit gesundes Verhalten zu erreichen (Foucault 1986, Martin 1993). Interventionen zielen nun entweder auf Körper oder auf materielles oder soziales Umfeld. Und da medikamentöse oder chirurgische Eingriffe nur in sehr speziellen Fällen greifen, werden für die Mehrheit der Übergewichtigen Interventionen propagiert, die über die Umwelt wirken. Dabei geht es nicht nur um die Bereitstellung eines gesünderen Angebots. Gesündere Lebensmittel in den Regalen, Spiel- 
und Sportplätze in den Städten und bezahlbare Vereinsangebote werden zwar als sinnvoll, häufig aber nicht mehr als ausreichend erachtet. Denn diese lassen den Einzelnen weiterhin die Wahl. Gemäßigte Positionen propagieren den Slogan: Die gesunde Wahl muss die einfachere sein. Dies ist vor allem in der Politik verbreitet, wo ein größeres Verständnis für die Rechtfertigung von Eingriffen in Privat- wie öffentliches Leben herrscht, als dies in der Biomedizin und Biotechnologie gemeinhin der Fall ist.

Wichtige Vertreter der Grundlagenforschung, sowohl Biologie wie Epidemiologie, sind mittlerweile allerdings dazu übergegangen, Maßnahmen zu fordern, die den Einzelnen keine Wahl mehr lassen. Wenn noch eine Wahl besteht, so die Argumentation, entscheiden sich viele doch wieder für die bequemere und damit ungesündere Alternative. Also müssen Rolltreppen durch Treppen ersetzt werden, müssen Lebensmittel selektiv so besteuert werden, dass ungesund $\mathrm{zu}$ teuer für viele wird, muss Schulsport ausgeweitet werden usw. In diesen Feldern vereinen sich nun zunehmend molekulare Verständnisse von Fett und Stoffwechsel, Erfahrungswissen aus klinischen Kontexten, Zynismus, Einsicht in die Trägheit politischer Prozesse und die Wirkmacht industrieller Interessen, zu einem präventiven Gefüge (Rabinow 2003, Deleuze/Guattari 1987), das zunehmend frühere Interventionen fordert. Kindergärten und Schulen sind die bevorzugten Ansatzpunkte. Denn wenn schlechte Gewohnheiten einmal Einzug gehalten haben, wenn Fettzellen, Stressantworten, Geschmacksvorlieben und Sportantipathien einmal in den Menschen installiert sind, so die Argumentation, dann wird das bisschen Willenskraft, das den meisten zur Verfügung steht, nicht reichen, um ein gesundes Leben zu führen.

Diese kurze Darstellung verdeutlicht, dass in den hier diskutierten Feldern vor allem über die Vermeidung risikobehafteter Lebensstile diskutiert wird. Häufig wird im Englischen von susceptibility gesprochen. Wir verwenden hier den Begriff Vulnerabilität. Diese beiden Konzepte - Risiko und Vulnerabilität - operieren nicht trennscharf. So, wie leicht erhöhter Blutdruck von Rosenberg als Protodisease bezeichnet wird, da er nicht im eigentlichen Sinne eine Krankheit darstellt, nun aber als solche behandelt wird, so könnte man von Vulnerabilität als Protorisiko sprechen (Rosenberg 2001). Vulnerabilität bezeichnet ein empfänglich sein für bestimmte ungesunde Einflüsse und ist damit weniger ausgeprägt als ein Risikofaktor. Im Kontext von Optimierung setzt Rose Vulnerabilität von enhancement, also Verbes- 
serung, ab (Rose 2007: 17ff.). Dies ist im Kontext der neuen Genetik durchaus eine wichtige Unterscheidung. Im Zusammenhang von Herzkreislauferkrankungen und Übergewicht spielt zumindest in Deutschland enhancement überhaupt keine Rolle. >Besser als Normalgewicht< macht als Konzept wenig Sinn. Hinzu kommen in Deutschland historische Sensibilitäten, die allem, was auch nur in die Nähe von eugenischen Praktiken gerückt werden könnte, das Leben schwer machen. Herzkreislauferkrankungen sind hier also noch wesentlich stärker an Vermeidung von Krankheit als an Verbesserung des Lebens orientiert.

Die relevantere Unterscheidung in Bezug auf Risiko, Vulnerabilität und Optimierung ist daher in diesem Kontext eine andere. Gesundheitsvorsorge und Prävention haben sich in der herkömmlichen Form an einem optimalen Lebensstil orientiert, d.h. Ernährungs- und Bewegungsmenge und -zusammensetzung waren, wenn nicht universell, so doch an so allgemein gefassten Populationen orientiert, dass für alle dieselbe Maßgabe galt. Abweichungen von diesem Optimum wurden und werden immer verstanden als Kompromisse, die den Notwendigkeiten der konkreten Situation geschuldet sind. Der neue Vulnerabilitätsdiskurs tendiert in eine deutlich andere Richtung. Der optimale Lebensstil orientiert sich jetzt nicht mehr an einem universellen Optimum, sondern an den individuellen körperlichen Vorgaben, die durch evolutionäre, elterliche und frühkindliche Prägung sowie frühe Einflüsse des sozialen und materiellen Umfelds vorgegeben sind. Die Maßgabe ist daher nicht mehr: >Lebe dem allgemeinen Ideal entsprechend, um Dein Risiko zu minimieren.< Sonder vielmehr: >Passe Dein Leben Deinem Körper an, um nicht durch Abweichungen empfänglich für Krankheiten zu werden.< Dies ist ein fundamentaler Wandel in der Logik von Prävention und Gesundheitsfürsorge weg von einem populationsbezogenen Idealbild hin zu einer Ausrichtung des Individuums an seinen eigenen körperlichen Vorgaben; diese jedoch immer zu verstehen als »Natur modelliert an Kultur als Praxis« (Rabinow 1992).

Wir haben oben von Vulnerabilitätsdiskurs gesprochen und nicht von Praxis, da sich diese neue Orientierung zunächst einmal nur im wissenschaftlichen und biomedizinischen Diskurs durchzusetzen beginnt. Damit die Personalisierung oder zumindest Umorientierung in der Konstruktion von Populationen, die mit diesem Wandel einhergehen muss, greifen kann, braucht es diagnostische Verfahren, die in der klinischen Praxis und der Versorgung eingesetzt werden können. Es ist unwahrscheinlich, dass diese Verfahren auf genomischer Ebene ange- 
siedelt und auf die Allgemeinbevölkerung ausgerichtet sein werden. $\mathrm{Zu}$ viele Hindernisse stehen derzeit einer individualisierten oder personalisierten Medizin auf dieser Ebene im Wege. Eine wie auch immer geartete Personalisierung von Prävention ist in diesem Sinne also nicht zu erwarten. Eine Verschiebung des Diskurses jedoch scheint zumindest mittelfristig wahrscheinlich. Und diese wird, wenn auch noch so krude, eine weitere Somatisierung von Bildung, Lebensstil und Identität vorantreiben. Denn nur wer möglichst viel über seinen Körper weiß, kann sich >seiner selbst< entsprechend verhalten. Optimierung hat im Kontext von Herzkreislauferkrankungen bzw. allgemeiner chronischer, multifaktorieller Erkrankungen zwar viel mit einer Politik des Lebens und mit Vulnerabilität zu tun. Es geht allerdings in keiner Weise um enhancement, sondern um eine Optimierung der Anpassung der Lebensführung an den Körper. Die bisherigen Auswirkungen dieser Verschiebungen werden im Folgenden mit Blick auf Subjektivierungsprozesse diskutiert.

\section{SUBJEKTIVIERUNG}

Ein molekularisiertes Verständnis von Krankheit und Leben, sowie die optimale Anpassung des Lebensstils an die eigenen körperlichen Voraussetzungen sind also zwei wichtige Merkmale spätmoderner Gesundheitsvorsorge und Prävention im Bereich der chronischen Erkrankungen. Um die Auswirkungen eines solchen Verständnisses auf Prozesse von Subjektivierung und kollektive wie individuelle Selbstverständnisse $\mathrm{zu}$ verstehen, ist es allerdings nötig, diese Verständnisse nicht als Ergebnisse abstrakter, scheinbar globaler, faktischer Wissenschaft zu sehen, sondern vielmehr auf die resultierenden Praxen, d.h. auf Prävention und Gesundheitsvorsorge als ein spätmodernes Projekt zu schauen, welches weit über das Medizinsystem hinausreicht.

Foucault hat auf zwei zentrale Achsen biopolitischer Macht- und Kontrollinstrumente hingewiesen: die Disziplinierung des Individuums und die Regulierung von Bevölkerung (Foucault 1983). Beide Achsen stehen nach wie vor in einem intensiven Spannungsverhältnis. Die oben analysierten Prägungs- und Einbettungsdiskurse tragen dazu bei, dass sich das Verhältnis der beiden Achsen zugunsten der bevölkerungsbezogenen Ansätze verschiebt. Settings, d.h. durch spezifische Variablen verstandene Lebensräume und -welten, rücken in den Vor- 
dergrund. Das soziale und vor allem das materielle Umfeld von Bevölkerungsgruppen rückt mit Blick auf seine adipo- und leptogenen Eigenschaften in den Fokus, d.h. Umwelten werden als dick- oder dünnmachend untersucht. Ziel solcher Studien ist immer die gezielte Veränderung durch direkte Eingriffe oder indirekt über Änderungen der Planungsgrundlage für bestimmte Räume. Adipogene Umwelten sind gekennzeichnet durch ein Überangebot an kalorienreicher Nahrung, ein Unterangebot an Bewegungsmöglichkeiten, hohe Wohndichte und oft minderwertige Bausubstanz sowie häufig ein hohes Gewaltpotential bzw. Aggressivität im öffentlichen Raum (z.B. Swinburn, et al. 1999).

Wichtig an dieser Entwicklung ist zunächst das Verschwinden des Subjekts, des autonomen Individuums, aus diesem Diskurs. Wie bereits oben beschrieben liegt einem solchen Zugriff unter anderem die Annahme zugrunde, dass es dem Individuum sowieso nicht gelingt, ihr oder sein Leben hin zu einem gesünderen Lebensstil zu ändern. Hier liegt also kein gouvernementaler Regierungsstil vor. Es geht nicht oder nicht mehr um die Aktivierung des Subjekts. Rose schreibt:

»While both the wealthy and the poor had always engaged in a range of practices to maintain health, now the maintenance and promotion of personal hygiene, healthy child-rearing, the identification and treatment of illness became central to forms of self-management that authorities sought to inculcate into citizens and hence to their own hopes, fears, and anxieties. Over this period, those who were citizens of the advanced industrial societies of the West became committed to these norms of health and hygiene disseminated through the practices of state, medical and philanthropic authorities, as matters of their own self-maintenance and self-formation (see, for one of many historical accounts, Valverde 1991). By the second half of the twentieth century, health had become one of the key ethical values in such societies. A plethora of medical and philantropic organisation engaged in campaigns of health education and health promotion, and made demands on political authorities in the name of health. Additionally, actual or potential patients and their families and advocates, now became key actors in the economics, politics, and ethics of health. ... Health, understood as an imperative, for the self and for others, to maximize the vital forces and potentialities of the living body, has become a key element in contemporary ethical regimes. «(Rose 2007: 22)

Diese Analyse trifft sicherlich auf viele Bereiche von Prävention und Gesundheitsvorsorge noch zu. Pädagogische, verhaltensorientierte und kognitive Ansätze der Habitualitisierung und Wissensvermittlung spielen immer noch eine zentrale Rolle, wie wir im Folgenden an ethno- 
graphischem Material noch diskutieren werden. Es zeichnet sich aber zumindest im deutschen Diskurs ein neuer Trend ab, der auf den ersten Blick eher an eine Hygienisierungsbewegung des ausgehenden 19. Jahrhunderts erinnert, als einen aktivierenden Diskurs des späten 20. Jahrhunderts.

Dies ist zum einen Entwicklungen in der Forschung und Fehlschlägen in bisherigen Präventionsbemühungen und damit verbundenen klinischen Erfahrungen geschuldet. Zum anderen hat in Deutschland in den letzten fünf Jahren eine starke Verknüpfung des Übergewichtsdiskurses mit einer neuen Unterschichts-Debatte stattgefunden. Diese neue Unterschicht hat wenig mit einer Arbeiterschicht zu tun, wie sie für ein Klassensystem englischer Provenienz herausgearbeitet worden ist.

»[Vielmehr] sieht [es] ganz so aus, als ob unsere Gesellschaft in ihrem Schoß das Profil einer Bevölkerungsgruppe wiederentdeckte, das man für verschwunden geglaubt hatte, >Nichtsnutze <, die sich darin aufhalten, ohne wirklich dazuzugehören. Ihnen kommt die Position von Überzähligen zu, die in einer Art gesellschaftlichem no man's land umhertreiben, die nicht integriert und zweifelsohne auch nicht integrierbar sind, zumindest in dem Sinne, in dem Durkheim von Integration als der Zugehörigkeit zu einer ein Ganzes bildenden Gesellschaft, bestehend aus voneinander abhängigen Teilen, spricht.« (Castel 2000: 359 zitiert in Lemke 2008: 104)

Analog zu dieser gesamtgesellschaftlichen Analyse lassen sich im Kontext von Prävention die >präventiven Nichtsnutze< als neue Subgruppe ausmachen, d.h. diejenigen Menschen, von denen eine präventive Medizin annimmt, dass sie sich in keiner Weise aktivieren lassen. Statistisch sind diese präventiven Nichtsnutze gekennzeichnet durch niedrigen sozio-ökonomischen Status, hohe Arbeitslosigkeit, Bildungsferne und hohen Migrationsanteil. Epidemiologisch findet sich eine hohe Prävalenz chronischer Erkrankungen, insgesamt erhöhte Mortalität und Morbidität, niedrigere Lebenserwartungen, erhöhter Anteil an Rauchern usw. Diese Charakteristika sind alle nicht neu. Neu wenigstens für europäische Gesellschaften hingegen ist der Ton, mit dem diese Menschen nun zunehmend >abgeschrieben< werden. Ihre Aktivierung wird als zunehmend aussichtslos beschrieben. Die Gründe für diese Aussichtslosigkeit werden entweder ethnisiert, d.h. auf kulturell verschiedene Ess- und Lebensgewohnheiten zurückgeführt, oder ökonomisiert, d.h. mangelnden Ressourcen und damit mangelnden 
Möglichkeiten und Interessen an Lebensgestaltung zugeschrieben (vgl. Fassin 2005).

Dabei geht die Verschärfung des Tons nicht in erster Linie auf die Wahrnehmung eines medizinischen Risikos zurück. Vielmehr wird diese neue >Unterschicht $<$ nun im politischen Diskurs als Bedrohung der Stabilität der Sozialsysteme dargestellt. Präventive Nichtsnutze werden früh chronisch krank und damit erwerbsunfähig bzw. nur eingeschränkt erwerbsfähig. Übergewicht ist eine sichtbare Manifestation dieses Problems. Die Sozial- und Gesundheitskassen belastet dies doppelt: zum einen über die Behandlungskosten, die gerade bei chronischen Erkrankungen, die durchgehend medikamentiert werden, hoch liegen; zum anderen über das fehlende Steuer- und Abgabenaufkommen. Das Verhältnis Einzahler zu Nutzer verschiebt sich in ein kritisches Ungleichgewicht - so jedenfalls die ökonomisch-medizinische Erzählung. Übergewicht und chronische Herzkreislauferkrankungen sind damit nicht mehr Privatangelegenheit, sondern stellen eine gesellschaftliche Belastung dar. Als medizinisch-ökonomisches Risiko werden Herzkreislauferkrankungen somit zum Politikum. Zu ihrer Bekämpfung formiert sich eine präventive Assemblage aus staatlichen und privaten Akteuren. Diese präventive Assemblage verbindet ein durch Molekularisierung verändertes Körperbild mit politisch-ökonomischen Bedrohungsszenarien und selbstverständlich auch handfesten Marktinteressen, um neue Formen der Optimierung durchzusetzen und $\mathrm{zu}$ institutionalisieren.

Ähnlich wie Rose möchten wir uns hier nicht anmaßen, eine epochale Veränderung zu diagnostizieren. Schreiben >aus der Mitte< einer möglichen Entwicklung heraus macht es prinzipiell schwer, mögliche Signifikanzen zu entdecken und zu bewerten. Jedoch zeigt sich in der deutschen Präventions- und Gesundheitsvorsorgelandschaft ein Topos, der bisher in dieser Form nicht theoretisiert worden ist: spezifische Milieus fallen aus dem Aktivierungsdiskurs heraus. Es geht also gerade nicht um die vor allem durch eine feministische Kritik immer wieder hervorgehobene Neuverteilung von Verantwortung an Patientinnen oder Ratsuchende unter dem Deckmantel von Entscheidungsfreiheit und Wahlmöglichkeit (>choice $<$ ). Gerade im Gegenteil kommen die einzelnen Mitglieder der neuen Milieus als Subjekte überhaupt nicht mehr vor. In ihrer Behandlung als Subjekte und den damit verbundenen Wahlmöglichkeiten liegt ja gerade das neue Risiko: nicht für die 
Gesundheit der Einzelnen und auch nicht für die Bevölkerung, sondern für die Stabilität der Sozial- und Gesundheitskassen.

Selbstverständlich ist der Aktivierungsdiskurs nicht einfach verschwunden. Er existiert in seinen vielen Facetten und Praxisformen parallel. Diese neue Tendenz trägt jedoch eher Züge eines Neo-Hygienismus, denn eines Aktivierungsdiskurses. Hier geht es mehr um eine neue Form der pastoralen Herrschaft. Rose analysiert die dominante Form pastoraler Macht des ausgehenden 20. Jahrhunderts noch wie folgt:

»This is not the kind of pastoralism where a shepherd knows and directs the souls of confused or indecisive sheep. It entails a dynamic set of relations between the effects of those who council and those of the counseled. These new pastors of the soma espouse the ethical principles of informed consent, autonomy, voluntary action, and choice and nondirectiveness. (Rose 2007: 29)

$\mathrm{Zu}$ Beginn des 21. Jahrhunderts und im Kontext der chronischen Erkrankungen zeigt sich nun allerdings eine neue, alte Herrschaftsform. Zwar stellen informierte Zustimmung, Autonomie, Freiwilligkeit und Entscheidungsfreiheit immer noch die zentralen medizinethischen wie politischen Werte dar. Jedoch ist eine Konfiguration im Entstehen begriffen, die durch zwei Phänomene gekennzeichnet ist, die bereits zum ersten Mal in der sozialen Hygiene und der Lebensreformbewegung Ende des 19. Anfang des 20. Jahrhunderts eine erste Blüte erlebten. Erstens verschränken sich etablierte, machtvolle und durchsetzungsstarke Institutionen und Diskurse beinahe lückenlos ineinander: medizinisches und ökonomisches Risiko gehen Hand in Hand und kommen zu einer gemeinsamen Problemdefinition. Zweitens gelingt es mittels verschiedener Wissensformen aus Medizin, Sozialarbeit, Pädagogik und Ökonomie, spezifische Milieus als Sonderfälle zu deklarieren, die besonderer Aufmerksamkeit und Hilfe bedürfen. Das Konzept der >aufsuchenden Prävention<, d.h. der Prävention, die nicht mehr als freiwilliges Angebot, sondern als gerichtete Maßnahme den als betroffen Identifizierten angetragen wird, steht hier exemplarisch für diesen neuen Ton. Diese Kombination aus wirkmächtiger Assemblage und Identifizierung einer Gruppe von Menschen, denen man eine besondere Behandlung zukommen lassen kann, treibt eine Tendenz zur Entmündigung spezifischer Milieus voran. Es entwickelt sich eine entsubjektivierte Präventionspraxis, die Zielgruppen ausmacht und dann für 
diese Entscheidungen im Sinne einer gesellschaftlichen Risikominimierung trifft.

Dies ist ohne Zweifel eine ambivalente Entwicklung, die sowohl biopolitische Repression wie post-liberalen Paternalismus möglich scheinen lässt. Allerdings schließen wir uns Rose und anderen auch in ihrer Skepsis an, dass es sich hier, wie von vielen schnell proklamiert, um eine neue Form der Eugenik handelt. Zunächst einmal geht es nicht in relevanter Weise um Reproduktionsentscheidungen. Zwar ist die neue Unterschichtendebatte auch mit der Frage um Geburtenpolitik verknüpft. Dies steht allerdings nur noch in einem sehr losen Bezug zu Präventionspolitik. Über Demographie und die Stabilität der Sozialsysteme lässt sich diese Brücke zwar schlagen, aber dies geschieht momentan nicht und ist auch nicht das vordringlichste Problem. Des Weiteren stehen im Zentrum der Präventionsdebatte nicht die Kernwerte von eugenischen Programmen: Nation, Qualität, Staatsgebiet, Population (und ggf. Rasse). Es geht auch in keiner Weise um die Fitness einer Nation in einem geopolitischen Wettstreit. Wenn überhaupt Fitness, dann steht die politische Fitness auf dem Spiel, mittels Gesundheitspolitik erfolgreich sozialstaatliche Stabilität $\mathrm{zu}$ sichern und Wachstum zu befördern. Zentral ist vielmehr die Frage nach dem >Wie< von Klassifikation und der Konstruktion von problematischen Subpopulationen.

Von einer Neoeugenik zu sprechen, verfehlt also das Ziel. Sinnvoller erscheint eine engmaschigere Untersuchung und Kritik der neuen Formen und Auswirkungen des präventiven Gefüges in verschiedenen Praxisfeldern. Denn es ist keineswegs davon auszugehen, dass biopolitische Regulierungsmaßnahmen, die von diesem präventiven Gefüge ausgehen, ohne Widerstand in die Praxis umgesetzt werden können. Wir zeigen im nächsten Abschnitt an einer kurzen ethnographischen Sequenz aus einem Berliner Kindergarten, dass Prävention nicht einfach auf ein unbestelltes Feld ausgetragen wird. Vielmehr entstehen eine Reihe von Ambivalenzen, wenn präventivmedizinisches Wissen auf etablierte Praxisformen trifft. 


\section{Prävention im Kindergarten}

Im Folgenden analysieren wir in Kürze anhand ethnographischen Materials die Auswirkungen des bisher skizzierten Dreischritts - Molekularisierung, Optimierung, Subjektivierung - auf Alltagspraxis am Beispiel von Kindergärten. Allein die Tatsache, dass Kindergärten in den letzten Jahren vermehrt Ziel von Präventionsprogrammen geworden sind, ist zum einen der Molekularisierung geschuldet: der eingebettete Körper muss geformt werden, bevor falsche Gewohnheiten sich somatisch manifestieren. Zum anderen, und dies verdeutlicht das Scheitern so vieler Präventionsprogramme mit Erwachsenen, kann dem erwachsenen Subjekt eine Anpassung seines Lebensstils an seinen Körper nicht mehr zugetraut werden. Die optimale Anpassung von Leben selbst an je spezifische körperliche Voraussetzungen muss also so früh wie möglich begonnen und von außen unterstützt werden.

Präventionsprogramme in Kindergärten produzieren daher nicht nur verschiedene Kinder >at risk< (Burri/Dumit 2007, Downey/Dumit 1997). Sie zielen vielmehr auf das gesamte >setting< Kindergarten und produzieren somit eine Konfiguration von Vulnerabilität und Prädisposition. Die Entwicklung von Kindern wird weniger als positive Bewegung in mögliche Zukünfte gesehen. Vielmehr bestimmt die Vermeidung negativer Szenarien die Konzepte von Entwicklung. Kognitive und behavioristische Ansätze, die Kinder als Individuen ansprechen, werden zunehmend durch setting Maßnahmen unterstützt, die Umwelten so gestalten, dass Entwicklungspfade >natürlicher Weise< und ohne individuelle Wahl- und Gestaltungsmöglichkeiten Vulnerabilitäten minimieren. Wir möchten an zwei Beispielen zum einen die Wirksamkeit dieser Präventionsprogramme illustrieren, zum anderen aber auch verdeutlichen, dass diese Programme nicht in einem Vakuum ansetzen, sondern bestehende Praxisformen $\mathrm{zu}$ transformieren versuchen. Dabei betont das erste Beispiel Optimierungsprozesse, wohingegen das zweite Beispiel eher Subjektivierung in den Vordergrund rückt.

Wie in vielen Kindergärten üblich so widmete sich auch einer der untersuchten Kindergärten im Dezember der Weihnachtsbäckerei. Es wurden Plätzchen gebacken. Jedoch war das Ergebnis keineswegs ein üppiger und festlicher Keksteller. Zwar hatten die Kinder vor dem Mittagsschlaf Dutzende von Ausstechern vorbereitet. Nachdem diese jedoch von den Betreuerinnen über Mittag gebacken worden waren, 
verschwanden alle bis auf einen pro Kind aus der Sicht- und Reichweite der Kinder. Beim Erwachen fand jedes Kind einen einzelnen Ausstecher auf seinem Teller drapiert.

Nun sind solche Aktivitäten als Rituale sowohl in ihrer ästhetischen als auch in ihrer edukativen Komponente bekannt und an sich nicht einem Präventionsprogramm zu verdanken. Signifikant in dieser Konstellation ist allerdings, dass die Betreuerinnen jedem Kind bereits einen und nur einen Keks auf den Teller legen. Hier spielt zum einen die materielle Dimension der Aktivität eine wichtige Rolle, denn mehr Kekse essen bedeutet auch mehr Zucker und Fett aufzunehmen. So findet biomedizinisches Wissen Eingang in Alltagspraxis. Zum anderen ist hier aber vor allem das weniger offensichtliche semiotische Arrangement von Bedeutung. Kinder erhalten weder Wahlmöglichkeiten mit Blick auf die Menge, die sie essen wollen. Ihnen wird aber auch nicht erlaubt, ihre Umwelt selbst zu gestalten. In vielen Kindergärten stehen ständig Wasser und Früchte zur freien Verfügung. Kekse jedoch werden hier strikt begrenzt und in Abwesenheit der Kinder während des Mittagsschlafs arrangiert. Kinder können daher >ungesunde< Lebensmittel immer nur ad limitum, nie aber ad libitum essen. Hier wird das Wesen des setting Ansatzes deutlich, der nicht primär auf Kinder >at risk< zielt, sondern Umwelten verändert, um so gesunde Gewohnheiten und Verhaltensweisen hervorzubringen. Kinder werden nicht als individuelle Akteure angesprochen. Statt der korporealen, hybriden, aktiven und politischen Aspekte jedes Kindes (Prout 1999, Burman 1994, Morss 1990), rücken Präventionsprogramme den Aspekt der vulnerablen Population in den Vordergrund.

Unser zweites Beispiel rückt allerdings solche Effekte von Präventionsprogrammen wie >Tigerkids $<$ in ein anderes Licht. Die Kontrolle von Essensquantität spielt eine wichtige Rolle in Kindergärten, die sich um Prävention in Sachen Ernährung und Bewegung bemühen. Die Hauptmahlzeiten spielen dabei selbstverständlich eine wichtige Rolle. In unserem Beispiel sitzen alle Kinder samt Betreuerinnen an einem Tisch. Kinder dürfen sich ihr Essen nur zu einem Teil selbst nehmen: in diesem Fall Nudeln. Warme und flüssige Speisen, d.h. Soße, werden von den Betreuerinnen serviert. Diese achten darauf, dass die erste Portion jedes Kindes möglichst klein gehalten ist, denn häufig möchten sich Kinder noch einmal nachnehmen. Möchte nun ein Kind eine zweite Portion, muss es danach fragen. Moritz (Pseudonym), das zweit dickste Kind der Gruppe, und der dünne Jan (Pseudonym) fragen 
gleichzeitig nach der Erlaubnis, sich eine zweite Portion nehmen zu dürfen. Die Betreuerin weicht zunächst aus, in dem sie den beiden sagt, dass sie nichts verstehen kann, wenn sie durcheinander reden. Jan soll als erstes sprechen. Jan fragt höflich nach einer zweiten Portion und bekommt $>$ Klar, nimm Dir doch was $<$ von der Betreuerin als Antwort. Danach fragt Moritz ebenso höflich. Die Betreuerin antwortet: »Ich weiß nicht, ob Du darfst. « Auf diese Weise lehnt sie die Verantwortung ab, Moritz eine zweite Portion zu verweigern. Eine strikte Weigerung würde höchstwahrscheinlich eine Beschwerde der Eltern nach sich ziehen und entspricht auch keineswegs dem Erziehungsverständnis der Betreuerinnen. Moritz soll selbst entscheiden.

In dieser kurzen Sequenz wird zunächst deutlich, dass setting Ansätze nie in Reinform auftreten bzw. auftreten können. Kindergärten als interaktive Räume können nur bedingt als gestaltbare Umwelten behandelt werden. Immer wieder ergeben sich fast notwendiger Weise Konstellationen, in denen Kindern Wahlmöglichkeiten gegeben werden. Dies führt dann häufig dazu, dass Konflikte zwischen institutionellen Zielen und kindlichen bzw. vor allem elterlichen Vorstellungen entstehen, die keineswegs in der Mehrzahl der Fälle zugunsten der Institution aufgelöst werden. Zwar gelingt es Betreuerinnen immer wieder, wie auch in unserem Beispiel, durch die Art wie Verantwortung an das Kind delegiert wird, dem übergewichtigen Kind ohne Verbot zu suggerieren, dass es wohl besser keine zweite Portion mehr essen sollte. Jedoch entstehen im Alltag viele Formen von Spannung und Widerständigkeit:

- In den Küchen: statt dem von Präventionsfachleuten erwünschten Fisch - um ein Beispiel zu nennen - werden Fischstäbchen serviert, da Kinder mit frischem Fisch heillos überfordert wären und die finanziellen Ressourcen weder ausreichen, um frischen Fisch einzukaufen, noch um genügend BetreuerInnen einzustellen, um alle Kinder beim Fischessen zu beaufsichtigen.

- In den Köpfen und Praxen der BetreuerInnen: Kinder sollen einerseits möglichst reibungslos dazu gebracht werden, sich gesund $\mathrm{zu}$ verhalten. Andererseits spielt aber auch die Erziehung zu eigenständigem Handeln eine zentrale Rolle in den Entwicklungskonzepten der BetreuerInnen. Was also tun, wenn Kinder sich eigenständig für ungesundes Verhalten entscheiden? 
- In den Elternhäusern: gerade in den von uns untersuchten Berliner Kindergärten weichen die stark kulturell geprägten Essgewohnheiten der Eltern deutlich von den Vorstellungen der deutschen BetreuerInnen ab. Vollkornbrot spielt nun einmal in türkischen und arabischen Haushalten keine Rolle. So sehen diese Eltern es häufig nicht gerne, dass Kindern in Kindergärten Vollkornbrot auch noch als die gesündere Alternative serviert wird.

Widerstand gegen verschiedene Elemente der Präventionsprogramme regt sich also auf materieller, professioneller und kultureller Ebene in der Alltagspraxis. Hier wird deutlich, dass Präventionsprogramme nicht in einem Vakuum ansetzen. Vielmehr greifen sie in hochgradig ausdifferenzierte und stabile Praxisformen ein. Selbstverständlich haben moderne westliche Erziehungs- und Entwicklungskonzepte auf holistische Art immer schon körperliche und geistige Komponenten mit unterschiedlichen Schwerpunktsetzungen berücksichtigt. Unter dem Einfluss der Roseschen Mutationen findet hier wieder eine Neukonfigurierung statt, die am ehesten als Re-Biologisierung von Entwicklung und Erziehung zu konzeptionalisieren ist (vgl. auch Morss 1990). Diese verläuft nicht konfliktfrei, denn anders als innerhalb der medizinischen Domäne, z.B. in Reha-Kliniken oder Gesundheitssporteinrichtungen, hat Medizin in dieser Konstellation keineswegs eine offensichtliche Deutungshoheit. Vielmehr müssen sich Präventionsprogramme gegen eine Menge unterschiedlicher Konzepte, pragmatischer Beschränkungen und kulturell geprägter Differenzen behaupten. Ihre Implementierung markiert daher immer einen Translationsprozess, im Rahmen dessen konkrete Vorgaben verändert und selektiert werden (vgl. Serres 1980/82, Serres 1983/91).

\section{SCHLUSSFOLGERUNGEN}

Der kurze ethnographische Exkurs verdeutlicht, dass Präventionspraxis immer schon anders ist, als in der Logik der Programme oder der präventiven Assemblage vorgesehen. Jede Praxisform aktualisiert ihre eigene Form von Prävention, so dass selbst aus einiger Distanz monolithisch erscheinende, nationale Präventionsprogramme wie Tigerkids im Alltag multiple Praktiken hervorbringen. Dies heißt natürlich nicht, dass politische Programme und medizinische Wahrheitsregime über- 
haupt keine Wirkmacht hätten. Sie verändern die Präventions- wie die Erziehungslandschaft. Aber auf die Frage, wie sie dies tun, bieten ethnographische Analysen eine wichtige Erweiterung an, die einen »modest empiricism« (Rabinow/Rose 2006) komplementieren.

Wir kommen letztlich zu dem Schluss, dass sich Herzkreislaufund Übergewichtsprävention sich von einer Technologie der Gesundheit zu einer Technologie des Leben selbst wandelt, so wie Franklin und Rose dies für die neue Genetik gezeigt haben. Allerdings wird der Dreischritt Molekularisierung, Optimierung und Subjektivierung nicht in allen Schritten nachvollzogen. Dies darf allerdings nicht als Kritik an der Roseschen Analyse verstanden werden, da Herzkreislauferkrankungen und Übergewicht als chronische, komplexe Krankheiten einen deutlich anderen Fall darstellen, als die von Rose, Rabinow, Franklin und anderen untersuchten direkten Anwendungen neuester genetischer Verfahren. Die ätiologischen Diskussionen um Herzkreislauferkrankungen erfahren eine deutliche Molekularisierung, die aber zunehmend das Konzept eines eingebetteten Körpers produziert. Dies unterscheidet Herzkreislauferkrankungen wesentlich von Gendiagnostik, Klonen oder Reproduktionstechnologien. Biologie wird hier also nicht verfügbarer, sondern im Gegenteil unverrückbarer und prägender. So kann man aus dem aktuellen Forschungsdiskurs zwar einen Trend zur Optimierung herauslesen; wobei Optimierung hier nicht im Sinne von enhancement, sondern im Sinne einer Anpassung von Leben selbst an seinen Körper verstanden werden muss. Daraus ergeben sich aber bisher keine neuen diagnostischen Verfahren oder Interventionen, die in direkter Weise auf Subjektivierungsprozesse wirken und damit Selbstverständnisse wie Verantwortungsmuster verschieben könnten. Vielmehr ergibt sich durch die Verknüpfung des medizinischen Diskurses mit der Wahrnehmung einer Bedrohung der Stabilität der Sozialsysteme durch lebensstilbedingte, chronische Erkrankungen eine Entsubjektivierung spezifischer Milieus, die dadurch Ziel einer wiederauflebenden, setting orientierten Prävention werden.

Der Aktivierungsdiskurs wird hier für spezifische Milieus ausgesetzt, da man den Mitgliedern dieser Milieus nicht zutraut, die Sorge für sich Selbst zu übernehmen. Die biopolitische Kritik an dieser Verschiebung haben wir in den vorangegangenen Abschnitten ausgeführt. Wir möchten an dieser Stelle zum Abschluss aber auch darauf hinweisen, dass pastorale Macht auch ihre guten Seiten haben kann. Das Konzept von >care<, d.h. von Achtsamkeit und sich kümmern, gewinnt 
in der Analyse von klinischen Praxen an Bedeutung (Mol 2008). Im Kontrast zu einem neoliberaleren Konzept von >choice<, erscheint der achtsame, vorsichtige und kümmernde Umgang von Ärzten mit ihren Patienten durchaus wünschenswert. Nach einer deutlichen Bewegung weg von paternalistischen Beziehungen hin $\mathrm{zu}$ shared decisionmaking, d.h. der zunehmenden Gleichberechtigung von informierten Patient_innen im Arzt-Patienten Verhältnis, stellt sich im Kontext von ärztlicher Versorgung nun die Frage nach einer neuen Logik der Achtsamkeit (>care< (Mol 2008)) und von verteilter Entscheidungsfindung (distributed decision-making (Rapley 2008)) bzw. Entscheidungsfindung als Prozess (vgl. Niewöhner 2010). Ähnliches scheint uns auf der Ebene von public health und Präventionsprogrammen zu gelten. Dabei geht es nicht um die Rechtfertigung einer Ausweitung medizinischer Deutungshoheit und Herrschaftskonstellationen. Es geht nicht um die etwas übergewichtigen Angehörigen einer breiten Mittelschicht. Vielmehr geht es darum ernstzunehmen, dass Ausgrenzung und Armut auch somatische Komponenten haben und diese direkt wie indirekt einen sehr realen Leidensdruck erzeugen. Es wäre also zu Fragen, ob der Kritik an pastoraler Macht nicht auch das positive Konzept einer pastoralen Verantwortung gegenübergestellt werden sollte. Ob und wie dies möglich sein wird, ohne in die paternalistischen Entwürfe des späten 19. und frühen 20. Jahrhunderts abzurutschen, wird für die Beobachter dieser Entwicklungen eine wichtige Frage und analytische Aufgabe darstellen. Erste hilfreiche konzeptionelle Schritte in diese Richtung geht zum einen eine alternative Lesart von Foucault, die sich die Untersuchung von Leben als solchem statt Leben selbst zum Ziel macht und damit auf Jacques Derrida und Walter Benjamin rekurriert, um die materiell und moralisch signifikanten Konsequenzen spezifischer Regierungstechniken für »Überleben« in den Blick zu nehmen. (Fassin 2009) Zum anderen spricht Vieles gegen eine Engführung des Konzepts der Politik des Lebens selbst und für eine Erweiterung hin zu multiplen Politiken des Lebens selbst. (Raman/Tutton 2009) Dieser Beitrag möchte zu dieser im Entstehen begriffenen Forschungsstrecke und damit zur Problematisierung und Entwicklung von Gouvernementalitätsstudien einen kleinen Beitrag geleistet haben. 


\section{LITERATUR}

Burman, E. (1994): Deconstructing Developmental Psychology, London/New York: Routledge.

Burri, R. V./Dumit, J. (2007): Biomedicine as Culture. Instrumental Practices, Technoscientific Knowledge, and New Modes of Life. New York: Routledge.

Clarke, A. E. et al. (2003): »Biomedicalization: Technoscientific Transformations of Health, Illness, and Us Biomedicine «. American Sociological Review 68, Nr. 2, S. 161-94.

Deleuze, G./Guattari, F. (1987): A Thousand Plateaus : Capitalism and Schizophrenia. Minneapolis: University of Minnesota Press.

Downey, G.L./Dumit, J. (1997): Cyborgs \& Citadels : Anthropological Interventions in Emerging Sciences and Technologies, Santa Fe/ NM.: School of American Research Press.

Fassin, D. (2005): »Compassion and Repression: The Moral Economy of Immigration Policies in France«. Cultural Anthropology 20 (3), S. 362-87.

Fisk, E. L. (1915): »The Prevention and Arrest of Diseases of the Cardio-Renal-Vascular System«. Am J Public Health 5 (12), S. 1214-23.

Foucault, M. (1983): Der Wille Zum Wissen, Frankfurt a.M.: Suhrkamp.

Foucault, M. (1974): Die Ordnung der Dinge. Eine Archäologie der Humanwissenschaften. Frankfurt/M: Fischer Taschenbuch.

Foucault, M. (1986): Die Sorge Um Sich, Frankfurt/M: Suhrkamp.

Franklin, S. (2000): »Life Itself: Global Nature and the Genetic Imaginary«. In: S. Franklin et al. (Hg.): Global Nature, Global Culture, London: Sage.

Gluckman, P. et al. (2005): »The Developmental Origins of Adult Disease «. Maternal \& Child Nutrition 1 (3), S. 130-41.

Hacking, I. (1983): Representing and Intervening: Introductory Topics in the Philosophy of Natural Science, Cambridge: Cambridge University Press.

Kahn, R. (2005): »The Metabolic Syndrome - Time for a Critical Appraisal«. The Lancet 366 (9501), S. 1921-22.

Kannel, W. B./McGee, D. L. (1979): »Diabetes and Cardiovascular Disease. The Framingham Study«. JAMA 241 (19), S. 2035-38. 
Keys, A. et al. (1950): »The Concentraion of Cholesterol in the Blood Serum of Normal Man and Its Relation to Age«, Journal of Clinical Investigation 29 (10), S. 1347-53.

Lemke, T: (2008): Gouvernementalität und Biopolitik, Wiesbaden: VS Verlag (2. Auflage).

Lengwiler, M./Beck, S. (2008): »Historizität, Materialität und Hybridität von Wissenspraxen. Die Entwicklung Europäischer Präventionsregime im 20. Jahrhundert«. Geschichte und Gesellschaft 34, S. 489-523.

Lengwiler, M./Madarász, J. (2010): Das Präventive Selbst. Eine Kulturgeschichte Moderner Gesundheitspolitik. Bielefeld: transcript.

Martin, E. (1993): »Histories of Immune Systems«. Culture, Medicine and Psychiatry 17 (1), S. 67-76.

McGowan, P. O. et al. (2009): »Epigenetic Regulation of the Glucocorticoid Receptor in Human Brain Associates with Childhood Abuse $\ll$. Nature Neuroscience (advanced online publication).

Mol, A. (2008): The Logic of Care. Health and the Problem of Patient Choice, London: Routledge.

Morss, J. (1990) The Biologising of Childhood: Developmental Psychology and the Darwinian Myth, Hove and East Sussex: Erlbaum.

Niewöhner, J. (2008): »Die Zeitlichen Dimensionen von Fett - Körperkonzepte zwischen Prägung und Lebensstil«. In: Niewöhner, J. et al. (Hg.):Wie Geht Kultur Unter Die Haut? Emergente Praxis Am Schnittfeld Von Medizin, Sozial- Und Lebenswissenschaften, Bielefeld: transcript, S. 111-40.

Niewöhner, J. (2010): »Entscheidungsprozesse in Der Hausärztlichen Übergewichtsberatung Aus Sozialanthropologischer Perspektive: Von Shared Decision-Making Zu Distributed Doctoring«. In: Heintze (Hg.): Public Health, Übergewicht Und Prävention, Weinheim: Juventa, S. 137-52.

Pickersgill, M. (2009): »Between Soma and Society: Neuroscience and the Ontology of Psychopathy«. BioSocieties 4, S. 45-60.

Prout, A. (Hg.) (1999): The Body, Childhood and Society, New York: St Martin's Press.

Rabinow, P. (2003): Anthropos Today. Reflections on Modern Equipment, Princeton: Princeton University Press.

Rabinow, P. (1992): »Artificiality and Enlightenment: From Sociobiology to Biosociality«. In: Crary, J./Kwinter, S. (Hg.): Incorporations, New York: MIT Press, S. 234-52. 
Rabinow, P./Rose, N. (2006): »Biopower Today«. Journal of BioSocieties 1 (2), S. 195-217.

Raman, S./Tutton, R. (2009): »Life, Science, and Biopower«. Science Technology Human Values 35, S. 711-34.

Rapley, T. (2008): »Distributed Decision Making: The Anatomy of Decisions-in-Action «. Sociology of Health \& Illness 30 (3), S. 429-44.

Reaven, GM. (1988): »Banting Lecture 1988. Role of Insulin Resistance in Human Disease «. Diabetes 37 (12), S. 1595-607.

Rose, N. (2007): The Politics of Life Itself, Princeton: Princeton University Press.

Roseboom, T. et al. (2006): »The Dutch Famine and Its Long-Term Consequences for Adult Health«. Early Human Development 82 (8), S. 485-91.

Rosenberg, C. E. (2001): »The New Enchantment: Genetics, Medicine, and Society in the Twenty-First Century«.

Roth, T. L. (2009): »Lasting Epigenetic Influence of Early-Life Adversity on the Bdnf Gene«, Biological Psychiatry (im Druck, Corrected Proof).

Serres, M. (1980/82): The Parasite. Baltimore, Maryland: Johns Hopkins University Press.

Serres, M. (1983/91): Rome. The Book of Foundations. Stanford/CA.: Stanford University Press.

Swinburn, B. et al. (1999): »Dissecting Obesogenic Environments: The Development and Application of a Framework for Identifying and Prioritizing Environmental Interventions for Obesity«. Preventive Medicine 29 (6), S. 563-70.

Szyf, M. (2008): »The Social Environment and the Epigenome«. Environmental and Molecular Mutagenesis 49 (1), S. 46-60.

Waterland, R.A. et al. (2004): »Early Nutrition, Epigenetic Changes at Transposons and Imprinted Genes, and Enhanced Susceptibility to Adult Chronic Diseases «. Nutrition 20 (1), S. 63-68.

Weaver, I.C.G (2004): »Epigenetic Programming by Maternal Behavior «. Nature Neuroscience 7 (8), S. 847-55. 